\title{
CONTRIBUIÇÃO AO ESTUDO DO COMPLEXO MÁFICO/ULTRAMÁFICO DE CAMPO FORMOSO USANDO SENSORIAMENTO REMOTO E DADOS AEROMAGNÉTICOS
}

\author{
RAIMUNDO ALMEIDA FILHO* , FRANCISCO J. F. FERREIRA** , FRANCISCO V. DA SILVA** \& PAULO VENEZIANI*
}

\begin{abstract}
CONTRIBUTION TO THE STUDY OF THE MAFIC/ULTRAMAFIC COMPLEX OF CAMPO FORMOSO, USINO REMOTE SENSING AND AEROMAGNETIC DATA The work discusses the combined use of remote sensing imagery and aeromagnetic data in the study of the Campo Formoso complex. The distribution of magnetic bodies associated with the mafic/ultramafic rocks of the complex shows strong NE-SW orientation, controlled by geologic structural features evident in satellite imagery. Structural analysis of the orbital imagery also suggests that the southern portion of the complex, where occur the main known chromium mineralization, constitutes a low structural block, better preserved by the erosion than the northern portion. Depth estimates of the top of magnetic sources based upon Euler's homogeneity equation, indicate that in the southern portion of the complex magnetic sources reach greater depth than in northern portion.

Key-words: remote sensing, magnetic data, mineral prospecting

RESUMO O trabalho discute o uso combinado de imagens de sensoriamento remoto e dados aeromagnéticos, no estudo do complexo Campo Formoso. A distribuicão dos corpos magnéticos associados às rochas máficas/ultramáficas do complexo reflete forte controle estrutural, segundo orientação geral NE-SW, evidenciado em imagens de satélite. Análise das imagens orbitais sugere também que o trecho sul do complexo, onde se localizam as principais mineralizações de cromo conhecidas, constitui um bloco estrutural rebaixado, melhor preservado pela erosão que o trecho norte, mais erodido. Estimativas de profundidades de topos de fontes magnéticas através de técnicas de deconvolução de Euler, indicam que na porção sul do complexo as fontes magnéticas atingem maiores profundidades que na porção norte.
\end{abstract}

Palavras-chaves: sensoriamento remoto, dados aeromagnéticos, prospecção mineral

INTRODUÇÃO O complexo máfico/ultramáfico de Campo de imagens de sensoriamento remoto orbital, como contribuição ao Formoso, no estado da Bahia (Figura 1), encerra as mais importantes mineralizações de cromo conhecidas no Brasil, as quais vêm sendo exploradas desde as primeiras décadas deste século. A despeito disso, os conhecimentos geológicos sobre o complexo são ainda limitados e na maioria dos casos restritos às áreas das minas de cromo. $\mathrm{O}$ mapeamento geológico de superfície é prejudicado pelo profundo intemperismo das rochas máficas/ultramáficas e pela presença de coberturas aluviais e coluviais, pouco conhecendo-se também sobre o comportamento do complexo em subsuperficie. Estudos sobre o comportamento espacial do complexo são de grande importância, visto tratar-se de um corpo estratiforme cujos níveis mineralizados conhecidos podem prolongar-se em profundidade.

Nesse contexto, o presente trabalho discute os resultados da análise de dados aeromagnéticos combinados com informações interpretadas

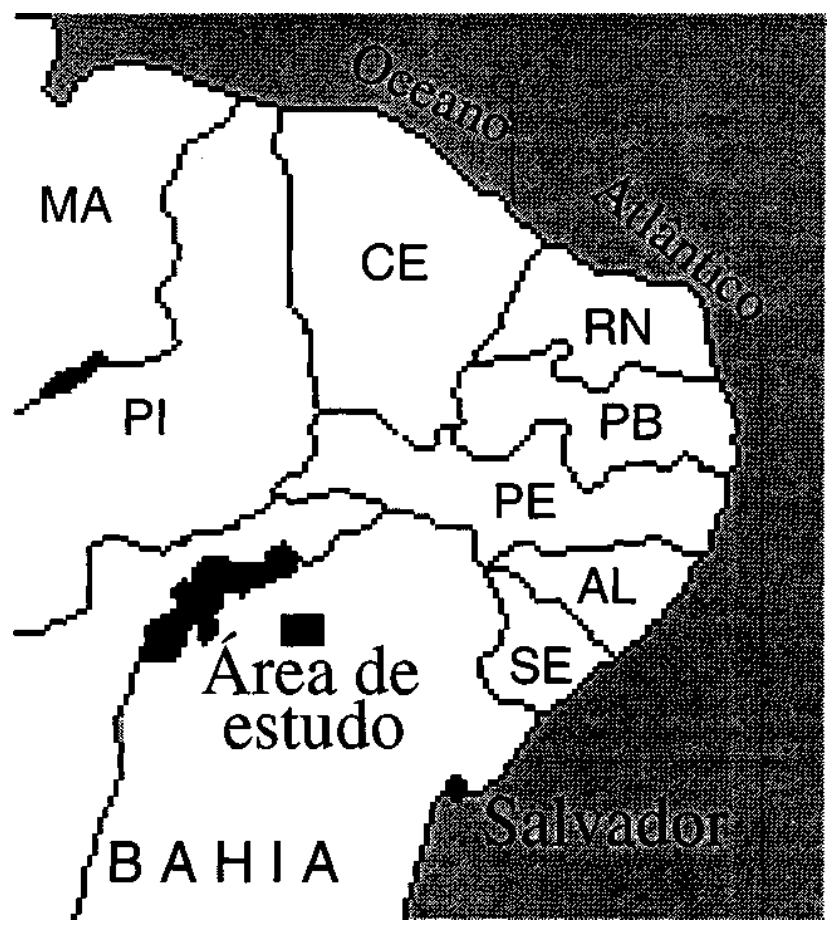

Figura l - Localização da área de estudo no estado da Bahia estudo do complexo Campo Formoso.

O complexo Campo Formoso, constituído por rochas máficas/ultramáficas do Proterozóico Inferior, ocorre como uma faixa alongada de direção geral NE-SW, com cerca de $40 \mathrm{~km}$ de extensão e larguras entre 100 e 1100 metros (Figura 2). Encontra-se alojado em rochas do embasamento de alto grau metamórfico (complexo metamórfico-migmatítico) e sotoposto às sequências metassedimentares do complexo Itapicuru, através da falha de Jacobina (Leo et al 1964), admitida como de caráter inverso. Suas relações de contato com o embasamento e com o batólito granítico de Campo Formoso não são claramente observadas, dado ao profundo intemperismo e à presença de coberturas aluviais e coluviais. A espessura original do complexo foi estimada por Thayer (1970) (in Gonçalves et al 1972), entre três e quatro mil metros, tendo sido erodidas suas porções superior e média.

Embora trate-se de um complexo estratiforme, a observação da continuidade de suas sequências em campo é dificultada devido o material original ter sido metamorfizado em diferentes graus, além de sofrer tectonismo, serpentinizacão e alteração intempérica. Dados do projeto Cromo de Campo Formoso (Gonçalves et al 1972) indicam uma sequência (da base para o topo) de actinolita gnaisse, tremolitaactinolita serpentinito e serpentina-clorita-carbonato-talco xistos, admitidos como derivados de peridotitos e piroxenitos. Estudos de Duarte \& Fontes (1986) nas minas Pedrinhas e Limoeiro, constataram a existência de mineralização em pelo menos sete níveis estratigráficos distintos com espessuras máximas de 12 metros.

MATERIAL E DADOS No estudo foram utilizadas imagens multiespectrais do Landsat-Thematic Mapper (TM), obtidas em 11 de fevereiro de 1991, sob ângulo de elevação solar de $49^{\circ}$, no formato digital e como composição colorida em papel, na escala $1: 250.000$. Foi usada também uma imagem de radar do satélite japonês JERS-1, em banda L, polarização $\mathrm{HH}$ e ângulo de incidência de $35^{\circ}$, obtida em 10 de agosto de 1994. As imagens (em formato digital) foram geometricamente corrigidas para a projeção Universal Transversa de Mercator (UTM), realçadas com linear constrast stretch e analisadas como bandas individuais ou composições coloridas falsa-cor. Os overlays da interpretação regional (imagem em papel), foram digitalizados e incorporados ao banco de dados. Nos processamentos digitais foi usado o Sistema de Processamento de Informações Georrenciadas SPRING, desenvolvido pelo Instituto Nacional de Pesquisas Espaciais (Câmara et al 1996). Feições lineares representadas por alinhamentos tonais, alinhamentos de drenagens, alinhamentos topográficos, ou combinações desses fatores, foram interpretadas no sentido proposto por O'Leary et al (1976), significando uma feição fotogeológica, retilínea ou levemente curvilinea, que presumivelmente reflete um fenómeno de subsuperficie, como fallhas/fraturas.

Por tratar-se de rochas máficas/ultramáficas ricas em minerais ferromagnéticos, o emprego de dados magnetométricos afigura-se como recomendável para o estudo do complexo. Esses dados foram

* Instituto Nacional de Pesquisas Espaciais-INPE, Caixa Postal 515, CEP 12227-010, São José dos Campos-SP, e-mail: rai@ltid.inpe.br

** UFPR - Departamento de Geologia, Caixa Postal 19011, CEP 81531-900, Curitiba-PR, e-mail: ferreira@setuva.geologia.ufpr.br 

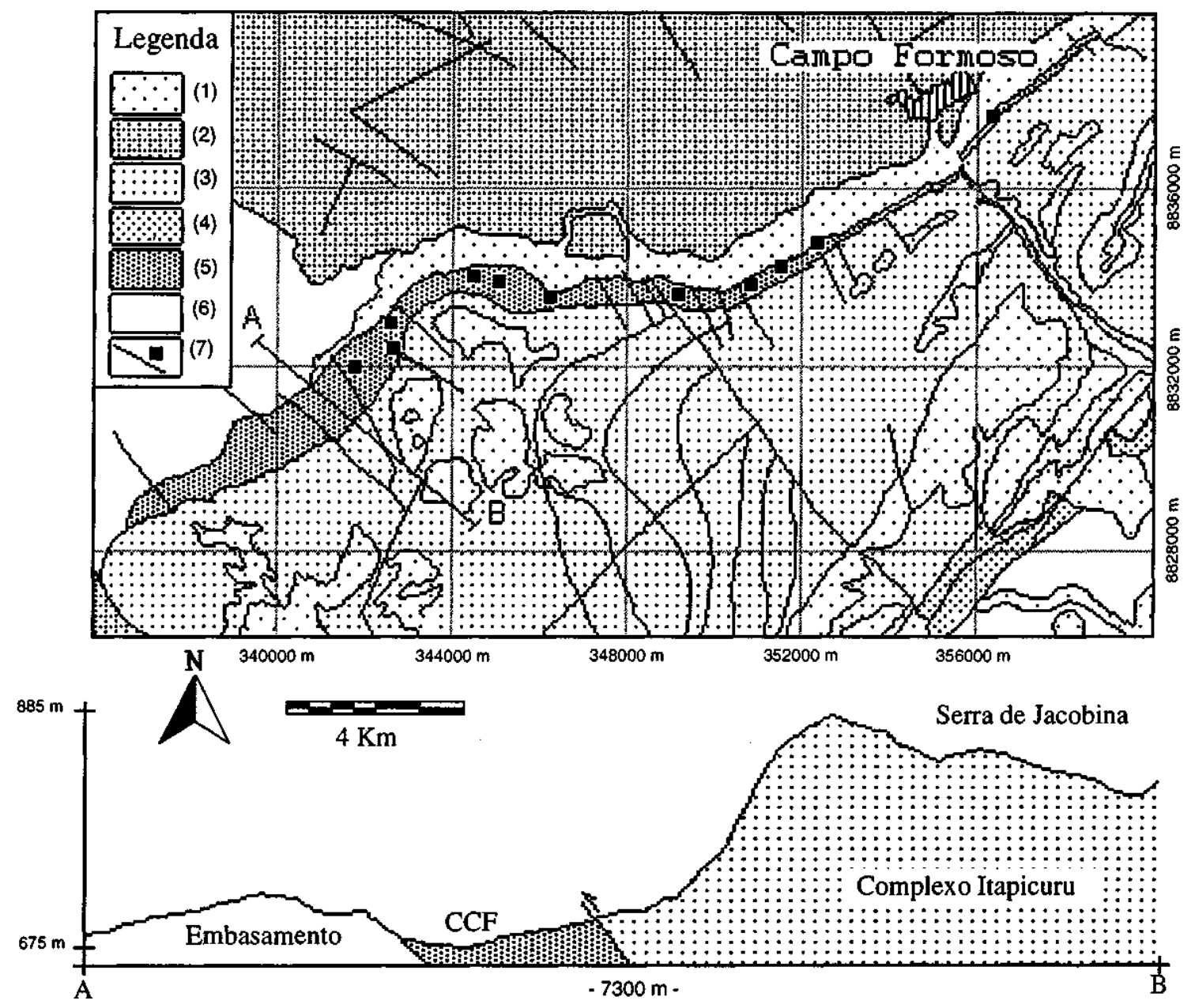

Figura 2. Mapa geológico generalizado da área de estudo (mod. de Couto et al. 1978): (1) depósitos aluviais e coluviais; (2) granitóides; (3) complexo Itapicuru; (4) complexo Saúde; (5) complexo Campo Formoso; (6) embasamento migmatítico; (7) falhas e mineralizações de cromo (Cascabulhos, Campinhos, Camarinha, Pedrinhas, Coitizeiro, Brejo Grande, Limoeiro, Sítio Angelim, Mato Limpo, Gameleira e Lava-Pés, da esquerda para a direita).

obtidos pelo projeto Serra de Itiúba (Anjos et al. 1980), no qual foi utilizado um magnetômetro modelo Geometrícs G-803, com precisão igual a $1 \mathrm{nT}$. A altura média de voo foi de 135 metros, segundo linhas com direção N45W espaçadas de $1 \mathrm{~km}$, com intervalo médio entre medidas de 110 metros. Para a geração do grid empregou-se um método bidirecional de interpolação, optando-se por uma grade regular de 125 metros (1/8 do espaçamento médio entre as linhas de vôo). Um interpolador linear foi usado ao longo das linhas de voo (em função da grande densidade de informação), enquanto um interpolador Akima spline foi aplicado na direção perpendicular. De modo a evitar a interferência de pequenas anomalias, aplicou-se um filtro tipo passa baixa, com frequência de corte igual ao inverso do dobro da dimensão da célula (frequência Nyquist $=0,004$ ciclos/metro), eliminando-se anomalias com comprimento de onda menor ou igual a 250 metros. Os dados foram corrigidos da variação diurna, subtraindo-se também o campo principal da Terra, representado pelo IGRF (International Geomagnetic Reference Field), correspondente ao ano de 1975. Mesmo instável em baixas latitudes magnéticas (no caso $\mathrm{I}=-12$ ), aplicou-se aos dados filtragem de redução ao Equador, de modo a centrar as anomalias diretamente sobre os corpos causadores dessas anomalias. Para processamento dos dados aeromagnéticos foram utilizados os pacotes Magmap e Gridepth (Geosoft 1994a , 1994b).

ANÁLISE DOS DADOS imagens de sensoriamento remoto De modo a situar a área de estudo no seu contexto regional, a interpretação das imagens orbitais na escala de 1:250.000 englobou uma área de aproximadamente $70 \mathrm{~km}$ de extensão entre as latitudes de $10^{\circ} 45^{\prime}-11^{\circ} 00^{\prime}$, ao longo do cinturão da Serra de Jacobina. Isso permitiu mapear estruturas geológicas regionais e caracterizar a natureza dos movimentos tectônicos definidos por essas estruturas. Evidências cinemáticas observadas nas imagens, tais como arrasto dúctil, rompimento e deslocamento de estratos e ou conjuntos litoestruturais, aliadas ao sentido de mergulho dessas unidades, permitiram supor que as feições identificadas tenham sido geradas em ambiente de cinturões de cavalgamento, definindo uma zona de cisalhamento com orientação geral NNE-SSW (Figura 3). Esse modelo de evolução estrutural é suportado também por evidências obtidas nos mapas geológicos do projeto Serra de Jacobina, como superposição de conjuntos litoestruturais mais antigos sobre conjuntos mais novos, além de sentido e ângulo de caimento de foliações medidas em campo.

A repetição de estratos e a geometria dos traços estruturais indicam a ocorrência de lascas de empurrões e ou cavalgamentos, delimitadas por zonas de cisalhamento rúptil a rúptil-dúctil, que caracterizam leques imbricados. Os mergulhos das lascas para SSE apresentam-se com ângulos em torno de $50^{\circ}$ a $60^{\circ}$, inferidos a partir de assimetrias de relevo e de drenagem e confirmadas por medidas de campo, indicando vergência para oeste e esforço máximo compressivo de este para oeste. As direcões estruturais regionais predominantes, orientadas segundo $\mathrm{N} 50-60 \mathrm{~W}$ e N50-60E, mostram movimentações com direções sinistrais e destrais oblíquas, respectivamente. O cruzamento dessas duas direcões (fora da área de estudo) define quatro cunhas com vértices negativos a norte e a sul e positivos a este e a oeste. Na área de estudo, a falha aqui denominado Rio do Aipim delimita porções estruturalmente soerguidas da cunha norte e rebaixadas da cunha sul (Figura 3). Essa configuração estrutural e a movimentação de blocos ao longo dessa falha são responsáveis pela inflexão de parte do cinturão de cavalgamentos (e do complexo Campo Formoso) que localmente assume direcão próxima a leste-oeste dentro da área de estudo. A falha Rio do Aipim parece prosseguir para além da área de estudo, sugerindo condicionar a bacia de sedimentação da Formação Bebedouro (Couto et. al., 1978) de idade pré-cambriana. 

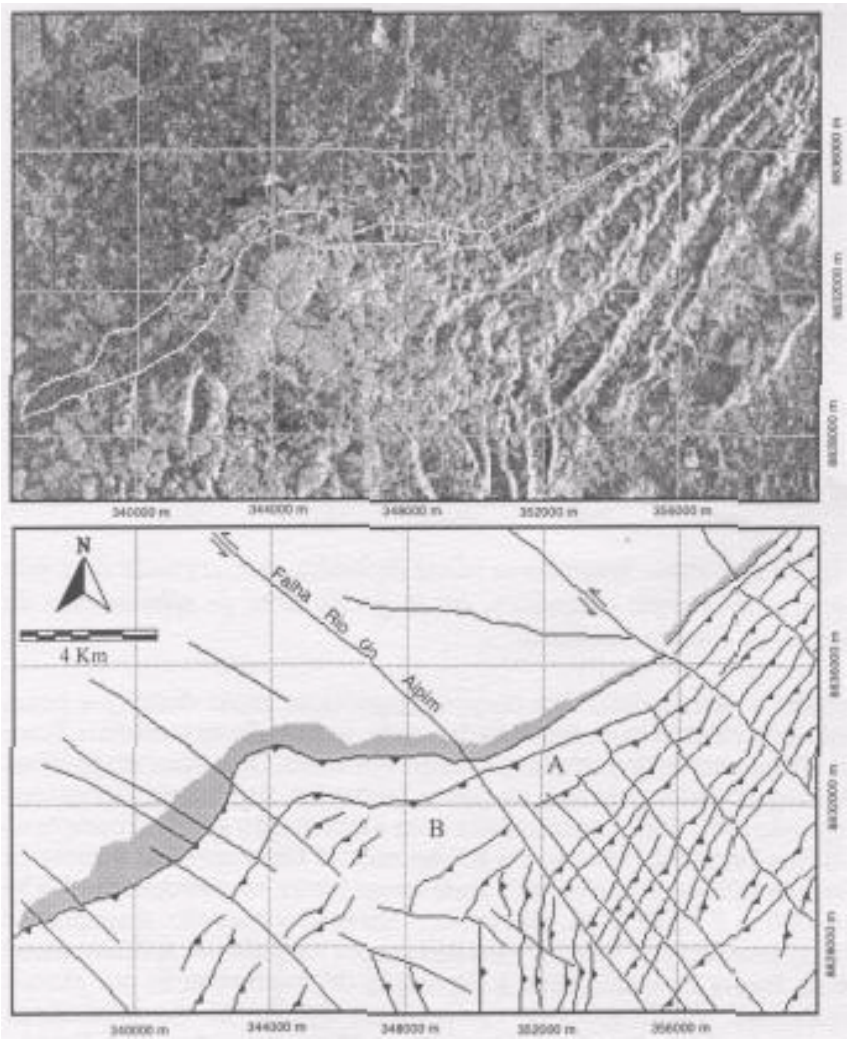

Figura 3. Imagem JERS/SAR cobrindo a área de estudo (a) e mapa estrutural interpretado com base em imagens orbitais (b).

Dados aerpmagnéticos DISTRIBUIÇÃO ESPACIAL DE FONTES $M A G N E T I C A S$ Técnica de deconvolução de Euler foi aplicada com o objetivo de definir a distribuição espacial das fontes magnéticas na área de estudo (rochas máficas/ultramáficas) e determinar a distribuicão estatística de profundidades de topos dessas fontes. A análise dos resultados obtidos foi feita à luz das informações geológicas disponíveis para a área de estudo, procurando-se verificar se feições geológicas conhecidas poderiam ser identificadas nesses dados; ou se feições geológicas sugeridas por eles poderiam ser melhor esclarecidas a partir de informacões extraídas de imagens de sensoriamento remoto. A técnica empregada tem como vantagens não necessitar o conhecimento da direção do vetor magnetização, nem a determinação do seu módulo, tornando a inversão um processo mais estável. Suas bases podem ser resumidas nas equações abaixo (Thompson 1982, Reid et al 1990). Uma função $(x, y, z)$ pode ser dita homogénea de grau $\mathrm{W}$ se $(t x, t y, t z)$ $=t^{n}(x, y, z)$ e sob essas condições, a assim denominada equação de Euler, pode ser redefinida como indicada em (7). Para uma fonte magnética, a mesma equação pode ser escrita como em (2), onde a posição do topo da fonte é dada por $\left(x_{o}, y o\right.$, Zo); Té o campo magnético medido em $(x, y, z)$ e $B$ um nível de base a ser determinado conjuntamente com a posição das fontes. $\mathrm{O}$ grau de homogeneidade $/ \mathrm{V}$ pode ser interpretado como um índice estrutural que indica as características geométricas da fonte. Considerando-se as características geológicas e estruturais do complexo Campo Formoso, adotou-se índice estrutural $N=$, por ser mais apropriado à definição de sills e diques irregulares (Reid et al 1990 ), admitindo-se $20 \%$ de tolerância de erro de profundidade das fontes.

$$
\begin{aligned}
& x \frac{\partial f}{\partial x}+y \frac{\partial f}{\partial y}+z \frac{\partial f}{\partial z}=n f \\
& \left(x-x_{0}\right) \frac{\partial T}{\partial x}+\left(y-y_{0}\right) \frac{\partial T}{\partial y}+\left(z-z_{0}\right) \frac{\partial T}{\partial z}=N\left(B-T_{1}\right.
\end{aligned}
$$

Os resultados foram plotados em três intervalos de profundidades de topos de fontes magnéticas, escolhidos arbitrariamente, a saber: 0$250 \mathrm{~m} ; 250-500 \mathrm{~m}$ e mais profundas que 500 metros. Na Figura 4 são indicados os mapas de distribuição espacial das fontes magnéticas
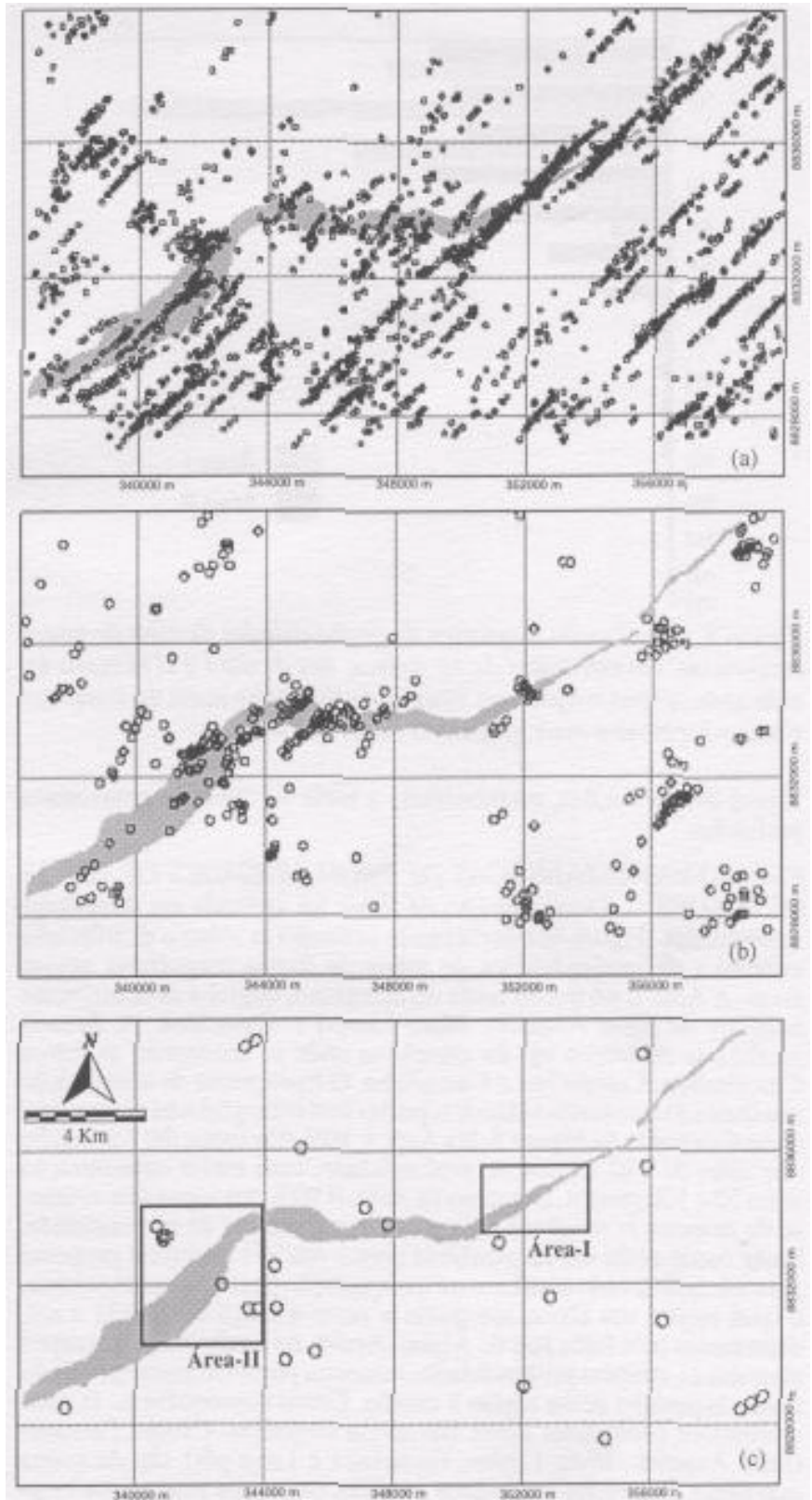

Figura 4. Distribuição espacial de fontes magnéticas na área de estudo, com base em técnicas de deconvolução de Euler, em três intervalos de profundidades de topos: 0-250 $\mathrm{m}$ (a), 250-500 $\mathrm{m}$ (b) $\mathrm{e}$ mais profundas que 500 metros (c).

nesses três intervalos de profundidades. A característica mais marcante observada é a forte orientação geral NE-SW, especialmente das fontes até 250 metros de profundidade e secundariamente até 500 metros. Comparando-se a distribuição espacial das fontes com o mapa estrutural obtido das imagens sensoriamento remoto (Figura 3), constata-se que a orientação das fontes magnéticas reflete as feições estruturais da área, moldadas pelas direções estruturais regionais orientadas segundo N50-60E.

Embora as fontes magnéticas orientem-se predominantemente segundo a direção NE-SW, elas são controladas também pelas direções estruturais N50-60W. Na Figura 4a é possível observar que o prosseguimento NE-SW das fontes é interrompido em certos locais por essas feições estruturais, especialmente na região sudoeste da área. A influência da direção estrutural noroeste é mais evidente nas fontes mais profundas, indicadas nas Figuras $4 \mathrm{~b}$ e $4 \mathrm{c}$. Nessa última figura as fontes são controladas essencialmente por essa direção estrutural, sugerindo a presença de falhas profundas. Couto et al (1978) mapearam corpos de metaultrabasitos tanto no complexo Itapicuru quanto no embasamento na região sudoeste da área, coincidentes com 


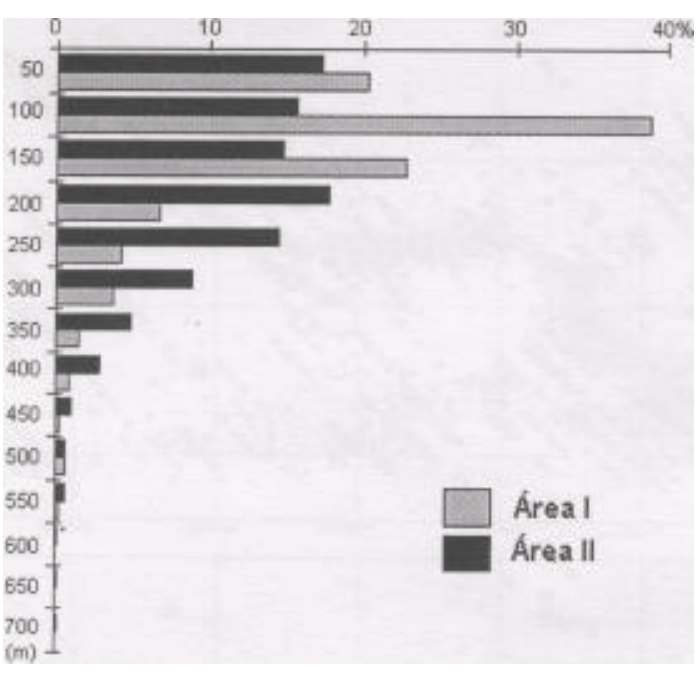

Figura 5. Distribuição estatística de profundidades de topo de fontes magnéticas, em intervalos de 50 metros, nas Áreas I e II (Figura 4), indicando corpos magnéticos mais rasos no trecho norte do complexo Campo Formoso e mais profundos no trecho sul.

alguns dessas feições, corroborando a ideia de tratar de geossuturas profundas.

ESTIMATIVAS ESTATÍSTICAS DE PROFUNDIDADES DE TOPOS $D E$ FONTES Deconvolução de Euler foi aplicada em duas áreas selecionadas (Figura 4c), fornecendo estimativas sobre a distribuição estatística de profundidades de topos de fontes magnéticas nessas áreas. A Área-I, no trecho norte do complexo, engloba as ocorrências minerais de Sítio Angelim, Mato Limpo e Gameleira. A Área-II localiza-se no trecho sul do complexo onde se encontram as minas Cascabulhos, Campinhos e Camarinha. O histograma de distribuicãa estatística das profundidades de topo das fontes magnéticas nessas duas áreas é indicado na Figura 5. Na Area-I, 80\% dos topos das fontes não vão além de 150 metros de profundidade, com maior concentração entre 50 e 100 metros, enquanto na Área-II 90\% dos topos distribuem-se de maneira homogénea até cerca de 300 metros de profundidade. Esses dados estão em consonância com o modelo estrutural proposto para a área de estudo com base na interpretação das imagens de satélite, o qual sugere um bloco soerguido a norte e outro rebaixado a sul, delimitados pela falha Rio do Aipim. Assim, no trecho norte os corpos magnéticos atingem profundidades menores, possivelmente refletindo maior exposição dessa região à erosão. Como consequência, as mineralizações conhecidas nesse trecho do complexo Campo Formoso (Sítio Angelim, Mato Limpo, Gameleira e Lava-pés) são de pouca expressão. No trecho sul, menos erodido, os corpos geológicos magnetizados (rochas máficas/ultramáficas) encontram-se melhor preservados e hospedam as principais minas de cromo conhecidas.

CORPOS MAGNÉTICOS RASOS Uma vez que a contribuição das fontes rasas é significativa em função da faixa aflorante de rochas máficas/ultramáficas, aplicou-se ao campo magnético residual reduzido ao equador um filtro de segunda derivada, com vistas a avaliar a distribuição dessas fontes. As curvas de valor zero definidas por essa função indicam, teoricamente, limites de fontes rasas, constituindo, desse modo, técnica útil em mapeamento geológico, dada à alta correlação com corpos geológicos magnéticos superficiais. A Figura 6

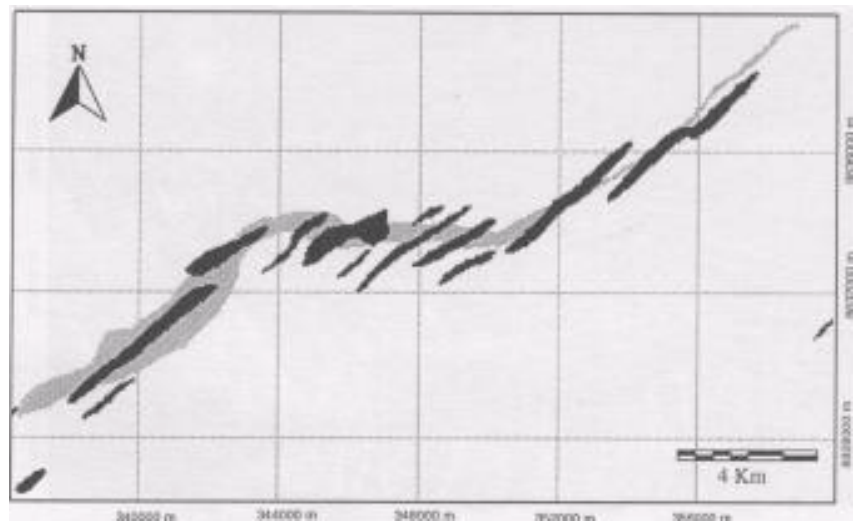

Figura 6. Corpos magnéticos rasos definidos pela segunda derivada vertical do campo magnético, ao longo da área de afloramento do complexo Campo Formoso.

mostra a distribuição dos corpos magnéticos rasos definidos pelas linhas de valor zero da segunda derivada, indicando-se também a faixa de afloramentos do complexo Campo Formoso. Os corpos magnéticos individualizados ocorrem na forma lenticular, fortemente orientados segundo N50-60E, concordantes com a orientação geral do complexo Campo Formoso. Mesmo no trecho onde a faixa máfica/ultramáfica assume orientação próxima a leste-oeste (entre as coordenadas UTM $344000 \mathrm{~m}$ e $350000 \mathrm{~m}$ ), eles conservam essa orientação, dispondo-se transversalmente à faixa. $\mathrm{O}$ fato dos corpos magnéticos apresentarem-se na forma de lentes, leva à suposição de fragmentação por efeitos tectônicos. Verificações de campo mais detalhadas são necessárias para esclarecer esse aspecto, que pode ser importante do ponto de vista prospectivo para cromo na área.

CONCLUSÕES O estudo permitiu delinear novas características sobre o comportamento espacial do complexo máfico/ultramáfico de Campo Formoso em superfície e subsuperfície. A distribuição regional das fontes magnéticas tem como característica marcante a forte orientação geral NE-SW, refletindo feições estruturais identificadas a partir da análise de imagens de sensoriamento remoto. Estimativas estatísticas de profundidade de topos de fontes magnéticas, combinadas com a análise das imagens, indicam corpos magnéticos mais rasos na porção norte do complexo e mais profundos no trecho sul, em blocos estruturais rebaixados a sul e soerguidos a norte. Ao longo da faixa máfica/ultramáfica os corpos mais magnéticos apresentam-se segmentados, formando lentes com orientação geral N50-60E. As constatações indicadas no estudo são importantes do ponto de vista prospectivo para cromo na área de estudo.

Agradecimentos À Fundação de Amparo à Pesquisa do Estado de São Paulo-FAPESP, ao Conselho Nacional de Desenvolvimento Científico e Tecnológico-CNPq, ao Instituto Nacional de Pesquisas Espaciais-INPE e ao Departamento de Geologia da Universidade Federal do Paraná, pelo suporte ao desenvolvimento do trabalho. Ao Earth Remote Sensing Data Analysis Center-ERSDAC, pelo fornecimento da imagem JERS/SAR; à Companhia de Pesquisa de Recursos Minerais-CPRM, pelo fornecimento dos dados aeromagnéticos; ao Departamento Nacional da Produção Mineral-DNPM, pelo fornecimento de relatórios e mapas dos projetos Cromo de Campo Formoso e Serra de Jacobina; e à Coitizeiro Mineração-COMISA, pelo apoio logístico durante visita à área.

\section{Referências}

Anjos, I.L S.; Mourão, L.M.F.; Marinho, F.A.V.; Mctelo, M.J.; Cardoso, C.E.T. 1980. Projeto Serra de Itiúha: relatório final. DNPM/CPRM, vol. 2, $18 \mathrm{p}$.

Câmara, G.; Souza, R.C.M.;Frcilas, U.M.; Garrido, J. 1996. SPRING: intcgratingremolcsensing and GIS by objecl-oriented data modcling. Comput. \& Graphic, 20:395-403.

Couto, P.A.; Gil, C.A.A.; Loureiro, H.C.; Arcanjo, J.B.; Fernandes Filho, J.; Guimarães, J.T.; Campeio, R.; Mascarcnhas, J.R.; Bruni, D.C.; Toledo, L.A.A.; Gonçalves, G.D. 1978. Projeto Serra de Jacobina: geologia e prospecção geoquímica. Relatório final. DNPM/CPRM, $347 \mathrm{p}$.

Duarte, P.M.; Fontes, C.F. 1986. Minas de cromita das fa/cndas Limoeiro c Pcdrinhas, município de Campo Formoso, Bahia. In: Schohbcnhaus, C.; Coelho, C.E.S. Principais depositos minerais do Brasil: ferro e metais da indústria do aço. DNPM/CVRD, vol. 2, cap. XVII, p. 207-216.

Gcosofl. 1994a. Magmap: 2-D frequency domain processing. Geosofl Inc. Toronto, 34 p. Geosoft.

1994b. Griclepth:3-D Euler deconvolution ofpotential datafield. Geosoft Inc. Toronto, $29 \mathrm{p}$

Gonçalves, J.C.V.; Moreira, J.F.C.; Hcdlund, D.C., Lima, P.E.; Quciro/, C.A. 1972. Projeto Cromo de Campo Formoso: relatório final. DNPM/CPRM, 181 p.
Leo, G.W.; COX, D.P.; Carvalho, J.P.P. 1964. Geologia da parte sul da Serra de Jacobina. Bahia, Brasil. DNPM/DGM, Boletim 209, 87 p.

O'Lcary, D.W.; Fricdman. J.D.; Pohn, H.A. 1976. Lincament, linear, lincation: some proposcd ncw standards for old tcrms. Geological Society of America Bulletin, 87:1463-1469.

Reid, A.B.; Allsop, J.M.; Granscr, H.; Millclt, A.J.; Somcrton, I.W. 1990. Magnctic interpretation in threc dimcnsions using Euler dcconvonlulion. Geophysics, 55: 80-91.

Thayer, T.P. 1970. Notes on the geology \& resources of someperidotites \& related rocks ofBra2.il. Mcmo rcporl USGS/AID (Rcport Bra/.ilian Invcsligalion - BR 32).

Thompson, D.T. 1982. EUDPH: A ncw tcchniquc for making computcr-assistcd depth cstimatcs from magnctic data. Geophysics, 47: 31-37. 